\title{
Antenna Calibration Technique Based on Channel Prediction in Time-varying TDD-MIMO System
}

\author{
Lijun Song ${ }^{\mathrm{a}}$, Lei Xia ${ }^{\mathrm{b}}$, Maozhu Jin ${ }^{\mathrm{c}, *}$ \\ ${ }^{a}$ Chengdu University of Information Technology, Chengdu 610225, P.R. China \\ ${ }^{b}$ University of Electronic Science and Technology of China,Chengdu, 610054, P.R. China \\ ${ }^{c}$ Business School of Sichuan University, Chengdu, 610065, P.R. China
}

\begin{abstract}
The traditional antenna calibration for time division duplex multiple input multiple output (TDD-MIMO) systems assume that free-space channel keeps constant during calibration, which is unreasonable under the high-speed rail and other time-varying channel scenarios for time variability can cause calibration error. This paper analyzes the performance of traditional antenna calibration methods, and then proposes an antenna calibration method based on Wiener channel prediction for time-varying TDD-MIMO system. Through theoretical derivation, we get the capacity formula of the TDD-MIMO system using traditional antenna calibration and antenna calibration based on channel prediction. The theoretical analysis and simulation results both indicate that the performance degradation of antenna calibration can be caused by time-varying channel and the prediction algorithm proposed in the paper can well compensate for the performance loss and significantly improve the antenna calibration performance
\end{abstract}

\footnotetext{
Fully documented templates are available in the elsarticle package on CTAN

* Corresponding author: Maozhu Jin,Business School of Sichuan University,Chengdu, 610065, P.R. China,

Email addresses: songlj@cuit.edu.cn (Lijun Song), leixia@uestc.edu.cn (Lei Xia), jinmaozhu@scu.edu.cn (Maozhu Jin)

${ }^{1}$ Lijun Song (b. July 3, 1975) is an instructor of college of communication engineering in Chengdu University of Information Technology. His research interests include OFDM and MIMO signal processing.

${ }^{2}$ Maozhu Jin (b. March 14, 1989) received his M.S. degree in the Department of Electronics Science and Technology and Ph.D. in the Department of Business from University of Huazhong Science and Technology, Wuhan, China, in 2005 and 2008, respectively. In April 2009, he joined Business School of Sichuan University, Chengdu, China. His research interests include multi-objective optimization, game theory, service science and supply chain management.
}

Preprint submitted to Journal of ${ }^{A} T_{E} X$ Templates

November 16, 2015

(C) 2015. This manuscript version is made available under the Elsevier user license http://www.elsevier.com/open-access/userlicense/1.0/ 
for time-varying TDD-MIMO system.

Keywords: Antenna calibration, Wiener channel prediction, Time-varying, MIMO

2010 MSC: 00-01, 99-00

\section{Introduction}

The mobile communications is undergoing the development process of evolution towards the broadband mobile communication, and the integration of the mobile communication technology and the broadband wireless access technology

5 continues to promote the development of the broadband mobile communications [1]-[3]. Therefore, the $3^{\text {rd }}$ Generation Partnership Project (3GPP) started the process of the long-term evolution for meeting the need of the broadband mobile communications development. It is noteworthy that the multipath effect and Inter Symbol Interference (ISI) caused by the multipath channel is more serious in broadband wireless communication. As an anti-multipath interference technology, the equalization can be realized at the transmitter side through pre-equalization or pre-coding technology. When equalized at the transmitter side, the transmitter should obtain channel information in advance from feedback information or channel reciprocity. In an ideal TDD system, as the downlink channel and uplink channel occupy the same frequency band, the transmitter can obtain downlink (uplink) channel state information (CSI) from uplink (downlink) channel measurements, i.e. channel reciprocity. Therefore, the transmitter in TDD systems can easily obtain CSI for equalization without any feedback information [4].

To date, the research on pre-equalization and pre-coding based on the channel reciprocity for TDD system is relatively mature. However, related studies of channel non-reciprocity still face some complicate problems, especially for the channel reciprocity loss caused by free-space channel and radio frequency $(\mathrm{RF})$ front-end. In practical communication system, the possible channel reci25 procity losses should be compensated before pre-equalization or pre-coding by 
using channel reciprocity, and this compensation technique is called antenna calibration[5][6][7]. Related studies have yielded some results. In [8] and [9], the response of analog devices was thought to be related with the external environment, such as temperature, power and time; models of the analog devices' response that change with environment parameters were established. A relative calibration that depends on a calibration phase to estimate the difference between uplink and downlink was proposed in [10][11]. In [12]-[16], authors analyzed the antenna calibration algorithm for MIMO and Coordinated Multiple Points (CoMP) systems, and obtained some valuable conclusions. However, the above methods assume that the free-space channel remains the same during calibration. When the speed of both communication sides are relatively low, the correlation of uplink (downlink) channels becomes strong due to weak time variability, and then the channel reciprocity can be used for pre-equalization or pre-coding in TDD systems. On the contrary, the uplink (downlink) chan40 nel correlation decreases and the properties of complete reciprocity is no longer established under the high-speed rail and other time-varying channel scenarios, resulting in loss of pre-equalization performance and channel reciprocity [17][18]. Therefore, the research on antenna calibration for time-varying TDDMIMO system has important theoretical value and practical significance.

45 Motivated by all of the above, this paper focuses on antenna calibration in the time-varying channels for TDD-MIMO system. A relative antenna calibration method based on Wiener channel prediction is proposed, where antenna calibration error due to time-variability channel can be reduced by channel prediction. Theoretical analysis and simulation results show that the proposed algorithm in this paper can well compensate for the performance loss and significantly improve the antenna calibration performance for time-varying TDDMIMO systems.

The rest of the paper is organized as follows. In Section 2, we describe the multi-antenna TDD pre-coding model and antenna calibration model for TDDMIMO system. Then we analyze the performance of the traditional antenna calibration and propose antenna calibration technology based on the Wiener 
prediction for time-varying TDD-MIMO systems in section 3. Simulation results are presented in Section 4. Finally, we conclude our work in Section 5.

\section{System model}

In this section, we describe the models of multi-antenna TDD pre-coding system and antenna calibration model for TDD-MIMO system.

\subsection{Multi-antenna TDD pre-coding system model}

Consider a multiple input multiple output orthogonal frequency division multiplexing (MIMO-OFDM) system with $N_{T}$ transmitter antennas, $N_{R}$ receiver antennas, and $N_{c}$ subcarriers. The received signal for the $t$-th OFDM symbol on the $k$-th $\left(k=1,2, \cdots, N_{c}\right)$ subcarrier can be expressed as [19]-[20]:

$$
\left[\begin{array}{c}
y_{1} \\
\vdots \\
y_{N_{R}}
\end{array}\right]_{(k, t)}=\left[\begin{array}{ccc}
h_{1,1} & \cdots & h_{1, N_{T}} \\
\vdots & \ddots & \vdots \\
h_{N_{R}, 1} & \ldots & h_{N_{R}, N_{T}}
\end{array}\right]_{(k, t)}\left[\begin{array}{c}
x_{1} \\
\vdots \\
x_{N_{T}}
\end{array}\right]_{(k, t)}+\left[\begin{array}{c}
n_{1} \\
\vdots \\
n_{N_{R}}
\end{array}\right]_{(k, t)}
$$

where $\mathbf{x}_{(k, t)} \in \mathbb{C}^{N_{T} \times 1}$ and $\mathbf{y}_{(k, t)} \in \mathbb{C}^{N_{R} \times 1}$ stand, respectively, for transmitting signal and received signal; $\mathbf{H}_{(k, t)} \in \mathbb{C}^{N_{R} \times N_{T}}$ and $\mathbf{n}_{(k, t)} \in \mathbb{C}^{N_{R} \times 1}$ are, respectively, channel matrix and white Gaussian noise. And the element $h_{i, j}$ of $\mathbf{H}_{(k, t)}$ denotes channel response from the $j$-th transmitter antenna of the base station to the $i$-th receiver antenna of the user. The received signal can be expressed in a vector form as

$$
\mathbf{y}_{(k, t)}=\mathbf{H}_{(k, t)} \mathbf{x}_{(k, t)}+\mathbf{n}_{(k, t)} .
$$

For simplicity, we remove index $t$ of OFDM symbols and index $k$ of subcarriers. Then we obtain

$$
\mathbf{y}=\mathbf{H x}+\mathbf{n} .
$$

According to the theory of singular value decomposition (SVD), matrix $\mathbf{H}$ can be expressed as

$$
\mathbf{H}=\mathbf{U D V}^{H},
$$


where $\mathbf{U}$ and $\mathbf{V}$ denote $N_{R}$ and $N_{T}$ order unitary matrix respectively.

$$
\mathbf{D}=\operatorname{diag}\left(\sigma_{1}, \sigma_{2}, \ldots, \sigma_{r}, 0, \ldots, 0\right)
$$

where $\sigma_{i}(i=1,2, \cdots, r)$ is the positive singular value of channel matrix $\mathbf{H}$. Therefore, $\mathbf{y}$ can be expressed as

$$
\mathbf{y}=\mathbf{U D V}^{H} \mathbf{x}+\mathbf{n} .
$$

Let

$$
\left\{\begin{array}{l}
\tilde{\mathbf{y}}=\mathbf{U}^{H} \mathbf{y} \\
\tilde{\mathbf{x}}=\mathbf{V}^{H} \mathbf{x} \\
\tilde{\mathbf{n}}=\mathbf{U}^{H} \mathbf{n}
\end{array}\right.
$$

According to the unitary matrix property $\mathbf{A}^{H} \mathbf{A}=\mathbf{I}$, Eq.(6) can be expressed as

$$
\tilde{\mathbf{y}}=\mathbf{D} \tilde{\mathbf{x}}+\tilde{\mathbf{n}} .
$$

Therefore, we can know that MIMO channel is equivalent to parallel subchannels through SVD. where $r=\operatorname{rank}(\mathbf{H}) \leq \min \left(N_{T}, N_{R}\right)$. i.e. [20]

$$
\tilde{y}_{i}=\sigma_{i} \tilde{x}_{i}+\tilde{n}_{i}, i=1,2, \ldots, r,
$$

where singular values indicate the channel gain of each sub-channel.

Using channel reciprocity, the transmitter at BS side obtain pre-coding matrix $\mathbf{V}_{U}$ by getting the singular value decomposition of $\mathbf{H}_{U}$ estimated from uplink channel, and then conduct the pre-coding on transmitting signal; the receiver at $\mathrm{UE}$ side obtain $\mathbf{U}_{D}$ by decomposition of $\mathbf{H}_{D}$ estimated from downlink, and then conduct decoding, and vice versa.

The input and output relationship of MIMO system using pre-coding is given by $[20]$

$$
\begin{array}{r}
\mathbf{y}=\mathbf{U}_{D}^{H}\left(\mathbf{H} \mathbf{V}_{U} \mathbf{x}+\mathbf{n}\right)=\mathbf{U}^{H}\left(\mathbf{U D V}^{H} \mathbf{V} \mathbf{x}+\mathbf{n}\right) \\
=\mathbf{U}^{H}(\mathbf{U D} \mathbf{x}+\mathbf{n})=\mathbf{U}^{H} \mathbf{U D} \mathbf{x}+\mathbf{U}^{H} \mathbf{n} \\
=\mathbf{D} \mathbf{x}+\tilde{\mathbf{n}}
\end{array}
$$

The average capacity of TDD-MIMO system based on SVD is [20]:

$$
C=\sum_{i=1}^{r} \log _{2}\left(1+\frac{P_{i}\left|D_{i, i}\right|^{2}}{\sigma_{n}^{2}}\right),
$$


where $P_{t}$ denotes transmission power of each antenna and it depends on the specific power allocation algorithm.

\subsection{Antenna calibration model for TDD-MIMO system}

The difference of RF front-end response lead to the equivalent channel reciprocity loss of each pair of antennas, i.e., equivalent uplink channel matrix $\tilde{\mathbf{H}}_{D}$ is no longer equal to $\tilde{\mathbf{H}}_{U}^{T}$, so Eq. (10) becomes[20]

$$
\begin{aligned}
\mathbf{y} & =\tilde{\mathbf{U}}_{D}^{H}\left(\tilde{\mathbf{H}}_{D} \mathbf{V}_{U} \mathbf{x}+\mathbf{n}\right) \\
& =\tilde{\mathbf{U}}_{D}^{H} \tilde{\mathbf{U}}_{D} \mathbf{D}_{D} \tilde{\mathbf{V}}_{D}^{H} \mathbf{V}_{U} \mathbf{x}+\tilde{\mathbf{U}}_{D}^{H} \mathbf{n} . \\
& =\mathbf{D}_{D} \tilde{\mathbf{V}}_{D}^{H} \mathbf{V}_{U} \mathbf{x}+\tilde{\mathbf{n}}
\end{aligned}
$$

Its indicated by Eq. (12) that the interference between data occurs and system capacity decreases. Therefore, it is quite necessary to use antenna calibration to compensate for the decreased system capacity caused by channel 75 reciprocity loss.

Fig.1 shows antenna calibration model for MIMO system [7]. In Fig.1, $g_{m, n}^{(U L)}$ and $g_{m, n}^{(U L)}$ are the free-space channel in uplink and downlink; $T_{B S, n}$ and $R_{B S, n}$ denote the transmitting RF front-end response and receiving RF front-end response at BS's $n$-th antenna, respectively, while $T_{U E, m}$ and $R_{U E, m}$ are the counterparts at UE's $m$-th antenna.

The channel reciprocity holds for free-space channel, i.e.

$$
g_{m, n}^{(D L)}=g_{m, n}^{(U L)}
$$

The measured uplink channel $h_{m, n}^{(U L)}$ and downlink channel $h_{m, n}^{(D L)}$ between UE's $m$-th antenna and BS's $n$-th antenna are expressed as

$$
\left\{\begin{array}{l}
h_{m, n}^{(U L)}=T_{U E, m} \cdot g_{m, n}^{(U L)} \cdot R_{B S, n} \\
h_{m, n}^{(D L)}=T_{B S, n} \cdot g_{m, n}^{(D L)} \cdot R_{U E, m}
\end{array}\right.
$$

We assume that $c_{B S, n}$ and $c_{U E, m}$ are, respectively, antenna calibration factor of the $n$-th antenna at BS and the $m$-th antenna at UE. $c_{B S, n}$ and $c_{U E, m}$ play an important role in compensating for mismatch of RF front-end response. 


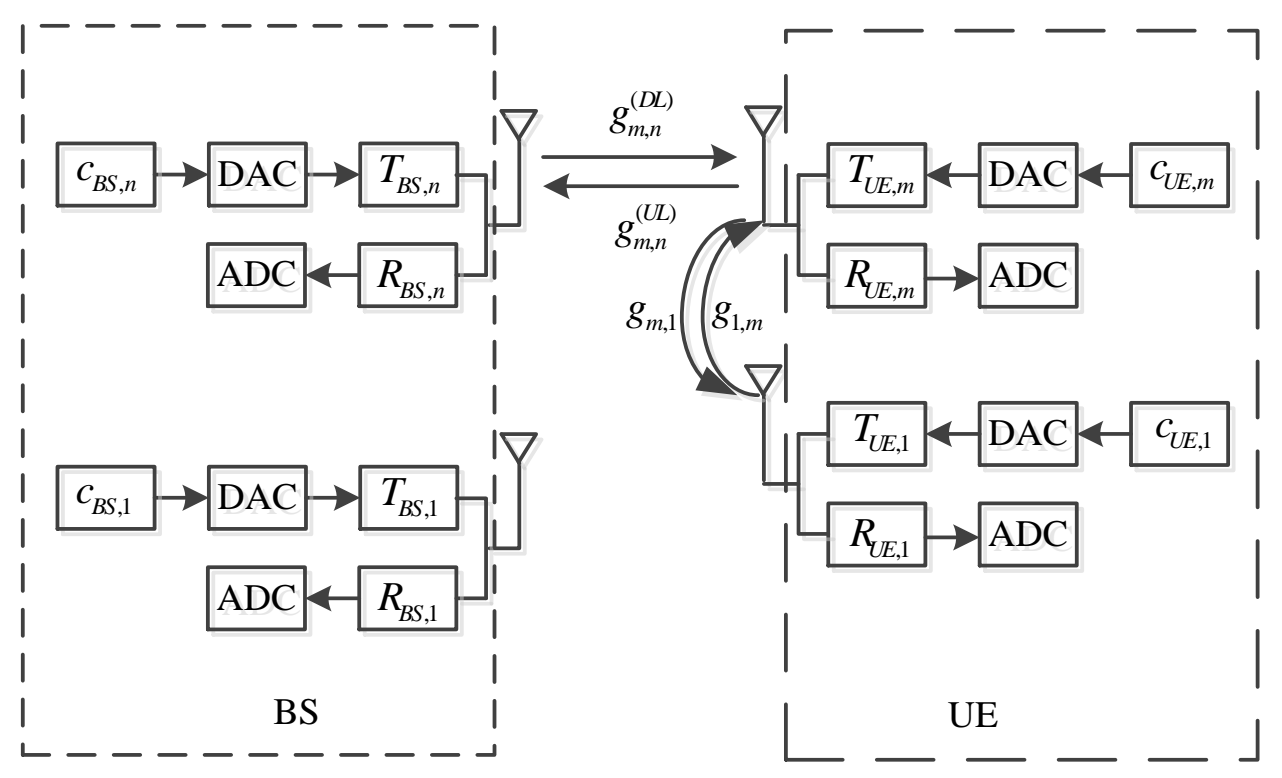

Figure 1: Antenna calibration model for MIMO system.

Therefore, the equivalent uplink and downlink channel after calibration can be expressed as

$$
\left\{\begin{array}{l}
a_{m, n}^{(U L)}=c_{U E, m} h_{m, n}^{(U L)} \\
a_{m, n}^{(D L)}=c_{B S, m} h_{m, n}^{(D L)}
\end{array}\right.
$$

The following conditions is required to exploit channel reciprocity, [9]

$$
\frac{a_{1,1}^{(U L)}}{a_{1,1}^{(D L)}}=\frac{a_{m, n}^{(U L)}}{a_{m, n}^{(D L)}} \quad m=1, \ldots, M \quad n=1, \ldots, N .
$$

Using Eq. (15), Eq. (16) is equivalent to

$$
\left\{\begin{array}{l}
\frac{c_{U E, 1} T_{U E, 1}}{R_{U E, 1}}=\frac{c_{U E, 2} T_{U E, 2}}{R_{U E, 2}}=\cdots=\frac{c_{U E, M} T_{U E, M}}{R_{U E, M}} \\
\frac{c_{B S, 1} T_{B S, 1}}{R_{B S, 1}}=\frac{c_{B S, 2} T_{B S, 2}}{R_{B S, 2}}=\cdots=\frac{c_{B S, N} T_{B S, N}}{R_{B S, N}}
\end{array}\right.
$$

Eq. (16) is also equivalent to

$$
\left\{\begin{array}{l}
\frac{a_{1,1}^{(U L)}}{a_{1,1}^{(D L)}}=\frac{a_{2,1}^{(U L)}}{a_{2}^{(D L)}}=\cdots=\frac{a_{M, 1}^{(U L)}}{a_{M, 1}^{(D L)}} \\
\frac{a_{1,1}^{(U L)}}{a_{1,1}^{(D L)}}=\frac{a_{1,2}^{(U L)}}{a_{1,2}^{(D L)}}=\cdots=\frac{a_{1, N}^{(U L)}}{a_{1, N}^{(D L)}}
\end{array}\right.
$$


Therefore, channel reciprocity in MIMO channel naturally holds when BS and UE perform antenna calibration individually in MISO channel.

UE determines $c_{U E, m}$ through mutual calibration as follow, [5]

1) BS estimates $h_{m, 1}^{(U L)}$ using calibration signals from UE and feeds back $h_{m, 1}^{(U L)}$

85 to $\mathrm{UE}(m=1, \ldots, M)$;

2) UE estimates $h_{m, 1}^{(D L)}$ using downlink calibration signals $(m=1, \ldots, M)$;

3) UE determines

$$
c_{U E, m}=h_{m, 1}^{(D L)} / h_{m, 1}^{(U L)} .
$$

The procedure of self-calibration is as follows [7]

1) Wet have known that

$$
h_{m 1, m 2}^{\left(U E \_s e l f\right)}=T_{U E, m 1} \cdot g_{m 1, m 2} \cdot R_{U E, m 2}
$$

is the equivalent channel response;

2) According to the condition of calibration, we have

$$
\frac{c_{U E, m 1}}{c_{U E, m 2}}=\frac{T_{U E, m 2}}{R_{U E, m 2}} \frac{R_{U E, m 1}}{T_{U E, m 1}}=\frac{h_{m 2, m 1}^{\left(U E_{\text {_s }} \text { elf }\right)}}{h_{m 1, m 2}^{\left(U E_{\text {s self }}\right)}} ;
$$

3) It is no problem in practice to set $c_{U E, m 1}=1$, other antennas' calibration factors can be easily computed.

Let $H_{m t}$ and $H_{m r}$ denote transmitting matrix and receiving matrix of UE' RF front-end, respectively, while $H_{b t}$ and $H_{b r}$ are the counterparts of BS's RF front-end. We have the following form of diagonal matrix

$$
\mathbf{H}_{b r}=\left[\begin{array}{ccc}
h_{b r, 1} & 0 & 0 \\
0 & \ldots & 0 \\
0 & 0 & h_{b r, N_{T}}
\end{array}\right],
$$

where $h_{b r, n}$ denotes the receiving gain at BS' $n$-th antenna. Diagonal elements can be expressed as

$$
h_{b r}=A_{b r} e^{j \phi_{b r}} .
$$

The antenna mismatch matrix is defined as follow

$$
\left\{\begin{array}{l}
\mathbf{E}_{b}=\mathbf{H}_{b t} \mathbf{H}_{b r}^{-1} \\
\mathbf{E}_{m}=\mathbf{H}_{m t} \mathbf{H}_{m r}^{-1}
\end{array}\right.
$$




\section{Antenna calibration based on Wiener channel prediction for multi- antenna system in time-varying channel}

In this section, we first analyze the performance of traditional antenna calibration for time-varying channel, and then propose an antenna calibration method based on Wiener channel prediction. Through theoretical derivation, we get the capacity formula of the system using traditional antenna calibration and antenna calibration based on channel prediction.

\subsection{Performance analysis of the antenna calibration technology based on multi-} antenna TDD pre-coding system for time-varying and the current equivalent uplink channel matrix, BS can obtain the calibration matrix when performing antenna calibration in time-varying channel. After calibration, both sides begin to transmit data. In order to research on performance loss caused by calibration error during calibration, we assume the uplink and downlink free-space channel is constant during data transmission, which can be applied by channel prediction technology.

Let the scenario in this section have one BS with two antennas and one UE with two antennas. For simplicity, we assume that UE's antennas have been calibrated and the transmitting and receiving gain of UE's antennas is 1.

Select an antenna of UE as reference, and then we perform antenna calibration between BS's two antennas and UE's reference antenna.

After calibration, BS uses SVD to pre-code and UE uses estimated downlink channel to decode. According to the assumption the free-space channel remains the same during data transmission, we have

$$
\mathbf{V}_{U}=\mathbf{V}_{D}
$$

Assuming that the channel is frequency flat fading channel, the signal after 
pre-coding can be expressed as

$$
\begin{aligned}
\mathbf{y} & =\mathbf{U}_{D}^{H}\left(\mathbf{H}_{D} \mathbf{I}_{i n} \mathbf{V}_{U} \mathbf{x}+\mathbf{n}\right) \\
& =\mathbf{U}_{D}^{H}\left(\mathbf{U}_{D} \mathbf{D}_{D} \mathbf{V}_{D}^{H} \mathbf{I}_{i n} \mathbf{V}_{U} \mathbf{x}+\mathbf{n}\right) \\
& =\mathbf{D}_{D} \mathbf{V}_{D}^{H} \mathbf{I}_{i n} \mathbf{V}_{D} \mathbf{x}+\tilde{\mathbf{n}}
\end{aligned}
$$

where $\mathbf{I}_{\text {in }}$ denotes interference matrix caused by channel time-variability during calibration. Considering that we only focus on antenna calibration at BS, $\mathbf{I}_{i n}$ can be expressed as

$$
\mathbf{I}_{i n}=\left[\begin{array}{cc}
\frac{h_{1}^{u}}{h_{1}^{d}} & 0 \\
0 & \frac{h_{2}^{u}}{h_{2}^{u}}
\end{array}\right],
$$

where $h_{1}^{u}$ and $h_{2}^{u}$ denote the uplink free-space channel response between BS' two antennas and UE' reference antenna; $h_{1}^{d}$ and $h_{2}^{d}$ denote the downlink freespace channel response between BS' two antennas and UE' reference antenna. In addition, $h_{1}^{u}$ and $h_{2}^{u}\left(h_{1}^{d}\right.$ and $\left.h_{2}^{d}\right)$ are independent of each other.

From Eq. (26), the gain matrix of received signal in time-varying channel can be expressed as

$$
\mathbf{E}=\mathbf{D}_{D} \mathbf{V}_{D}^{H} \mathbf{I}_{i n} \mathbf{V}_{D}
$$

Using Eq. (26) and Eq. (28), we have

$$
\begin{gathered}
\mathbf{y}=\mathbf{D}_{D} \mathbf{V}_{D}^{H} \mathbf{I}_{i n} \mathbf{V}_{D} \mathbf{x}+\tilde{\mathbf{n}} \\
=\mathbf{E x}+\tilde{\mathbf{n}}
\end{gathered}
$$

Similar to the situation of time-invariant channel, the average capacity of TDD-MIMO system based on SVD method for time-varying channel can be expressed as [18]

$$
C=\sum_{i=1}^{r} \log _{2}\left(1+\frac{P_{i}\left|e_{i, i}\right|^{2}}{\sum_{j=1, j \neq i}^{r}\left|e_{i, j}\right|^{2}+\sigma_{n}^{2}}\right),
$$

where $r=\operatorname{rank}\left(\mathbf{H}_{D}\right) \leq \min \left(N_{T}, N_{R}\right),\left\{e_{i, j}\right\}$ is the element of matrix $\mathbf{E}$ in Eq. (28); the diagonal elements of matrix $\mathbf{E}$ are the signal gain of parallel subchannels while the nonzero elements in non-diagonal of matrix $\mathbf{E}$ are interference 
between sub-channels; $\sigma_{n}^{2}$ denotes the variance of noise. Equal power allocation method is adopted here, i.e. $P_{i}=P_{T} / N_{T}$.

According to AR model, we can get [21][22]

$$
\begin{gathered}
h_{1}^{u}=J_{0}\left(2 \pi f_{d} \delta T_{s}\right) \cdot h_{1}^{d}+\sqrt{1-J_{0}^{2}\left(2 \pi f_{d} \delta T_{s}\right)} \cdot v_{1} \\
=\beta \cdot h_{1}^{d}+\sqrt{1-\beta^{2}} \cdot v_{1} \\
h_{2}^{u}=\beta \cdot h_{2}^{d}+\sqrt{1-\beta^{2}} \cdot v_{2}
\end{gathered}
$$

where $\beta=J_{0}\left(2 \pi f_{d} \delta T_{s}\right) ; v_{1}$ and $v_{2}$ denote the Gaussian variables with zero mean and unit variance.

Substituting Eq. (31), Eq. (32) and $\beta=J_{0}\left(2 \pi f_{d} \delta T_{s}\right)$ into Eq. (26), we obtain

$$
\begin{aligned}
\mathbf{y} & =\mathbf{D}_{D} \mathbf{V}_{D}^{H}\left[\begin{array}{cc}
\beta+\frac{\sqrt{1-\beta^{2}} v_{1}}{h_{1}^{d}} & 0 \\
0 & \beta+\frac{\sqrt{1-\beta^{2}} v_{2}}{h_{2}^{d}}
\end{array}\right] \mathbf{V}_{D} \mathbf{x}+\tilde{\mathbf{n}} \\
& =\mathbf{D}_{D} \mathbf{V}_{D}^{H} \beta\left[\begin{array}{cc}
1 & 0 \\
0 & 1
\end{array}\right] \mathbf{V}_{D} \mathbf{x}+\mathbf{D}_{D} \mathbf{V}_{D}^{H} \sqrt{1-\beta^{2}}\left[\begin{array}{cc}
\frac{v_{1}}{h_{1}^{d}} & 0 \\
0 & \frac{v_{2}}{h_{2}^{d}}
\end{array}\right] \mathbf{V}_{D} \mathbf{x}+\tilde{\mathbf{n}} \\
& =\beta \mathbf{D}_{D} \mathbf{x}+\sqrt{1-\beta^{2}} \mathbf{D}_{D} \mathbf{V}_{D}^{H}\left[\begin{array}{cc}
\frac{v_{1}}{h_{1}^{d}} & 0 \\
0 & \frac{v_{2}}{h_{2}^{d}}
\end{array}\right] \mathbf{V}_{D} \mathbf{x}+\tilde{\mathbf{n}}
\end{aligned}
$$

Assume

$$
\mathbf{V}_{D}=\left[\begin{array}{ll}
v_{11} & v_{12} \\
v_{21} & v_{22}
\end{array}\right]
$$

We define the expressions

$$
\begin{aligned}
& \left|\mathbf{V}_{D}\right| \wedge^{\wedge} 2=\left[\begin{array}{cc}
\left|v_{11}\right|^{2} & \left|v_{12}\right|^{2} \\
\left|v_{21}\right|^{2} & \left|v_{22}\right|^{2}
\end{array}\right], \\
& \left|\mathbf{D}_{D}\right| .^{\wedge} 2=\left[\begin{array}{ll}
\left|d_{11}\right|^{2} & \left|d_{12}\right|^{2} \\
\left|d_{21}\right|^{2} & \left|d_{22}\right|^{2}
\end{array}\right] .
\end{aligned}
$$

From Eq. (33) to Eq. (36), we can obtain signal power, interference power 
and noise power after lengthy but straightforward computation

$$
\begin{aligned}
& P_{s}=\beta^{2} \cdot\left|\mathbf{D}_{D}\right| \cdot{ }^{\wedge} 2+\left(1-\beta^{2}\right) \cdot E\left(\frac{1}{\left|h^{d}\right|^{2}}\right) *\left(\left|\mathbf{D}_{D}\right| \cdot{ }^{\wedge} 2\right) * \operatorname{diag}\left(\left(\left|\mathbf{V}_{D}^{H}\right| \cdot{ }^{\wedge} 2\right) *\left(\left|\mathbf{V}_{D}\right| \cdot{ }^{\wedge} 2\right)\right), \\
& P_{i n}=\left(1-\beta^{2}\right) \cdot E\left(\frac{1}{\left|h^{d}\right|^{2}}\right) \cdot\left(\left|\mathbf{D}_{D}\right| \cdot{ }^{\wedge} 2\right) *\left(\left(\left|\mathbf{V}_{D}^{H}\right| \cdot{ }^{\wedge} 2\right) *\left(\left|\mathbf{V}_{D}\right| \cdot{ }^{\wedge} 2\right)-\operatorname{diag}\left(\left(\left|\mathbf{V}_{D}^{H}\right| \cdot{ }^{\wedge} 2\right) *\left(\left|\mathbf{V}_{D}\right| \cdot{ }^{\wedge} 2\right)\right)\right),
\end{aligned}
$$

$$
P_{n}=\sigma_{n}^{2}
$$

where

$$
E\left(\frac{1}{\left|h_{1}^{d}\right|}\right)=E\left(\frac{1}{\left|h_{2}^{d}\right|}\right)=\int_{v_{0}}^{\infty} \frac{1}{v} e^{-v} d v .
$$

Through rigorous analysis, we know that Eq. (37), Eq. (38) and Eq. (39) are corresponding to $\left|e_{i, i}\right|^{2},\left|e_{i, j}\right|^{2}$ and $\sigma_{n}^{2}$ in Eq. (30). Substituting Eq. (37),

125 Eq. (38) and Eq. (39) into Eq. (30), we can obtain the system capacity formula after using the antenna calibration algorithm in time-varying channel.

We normalize the integral in Eq. (40), we have

$$
E\left(\frac{1}{\left|h_{1}^{d}\right|}\right)=E\left(\frac{1}{\left|h_{2}^{d}\right|}\right)=\int_{v_{0}}^{\infty} \frac{1}{v} e^{-v} d v=1,
$$

$v_{0}$ can be computed as 0.265 .

\subsection{Antenna calibration technology based on Wiener channel prediction in MI-} MO system

Similar with the channel prediction process of single-antenna calibration [23], BS obtains equivalent downlink channel response fed back by UE, and then conducts Wiener channel prediction [21] to achieve the channel response for uplink transmitting signal. In addition, we can obtain equivalent uplink channel response estimated by BS. Therefore, calibration factors between antenna pairs can be computed. 
After prediction, the received signal can be expressed as

$$
\begin{aligned}
\mathbf{y} & =\mathbf{D}_{D} \mathbf{V}_{D}^{H}\left[\begin{array}{cc}
\frac{h_{1}^{u}}{\widehat{h}_{1}^{u}} & 0 \\
0 & \frac{h_{2}^{u}}{\widehat{h}_{2}^{u}}
\end{array}\right] \mathbf{V}_{D} \mathbf{x}+\tilde{\mathbf{n}}=\mathbf{D}_{D} \mathbf{V}_{D}^{H}\left[\begin{array}{cc}
1+\frac{\delta_{1}}{\widehat{h}_{1}^{u}} & 0 \\
0 & 1+\frac{\delta_{2}}{\widehat{h}_{2}^{u}}
\end{array}\right] \mathbf{V}_{D} \mathbf{x}+\tilde{\mathbf{n}} \\
& =\mathbf{D}_{D} \mathbf{x}+\mathbf{D}_{D} \mathbf{V}_{D}^{H}\left[\begin{array}{cc}
\frac{\delta_{1}}{\widehat{h}_{1}^{u}} & 0 \\
0 & \frac{\delta_{2}}{\widehat{h}_{2}^{u}}
\end{array}\right] \mathbf{V}_{D} \mathbf{x}+\tilde{\mathbf{n}}
\end{aligned}
$$

where $\hat{h}_{1}^{u}$ and $\hat{h}_{2}^{u}$ are uplink channel response predicted by M downlink channel response $\mathbf{h}_{1}^{d}$ and $\mathbf{h}_{2}^{d}$, respectively. After prediction, the real uplink channel response can be expressed as

$$
\left\{\begin{array}{l}
h_{1}^{u}=\hat{h}_{1}^{u}+\delta_{1} \\
h_{2}^{u}=\hat{h}_{2}^{u}+\delta_{2}
\end{array}\right.
$$

In addition, $\sigma_{\delta_{1}}^{2}=\sigma_{\delta_{2}}^{2}=J_{\min }$.

After the similar derivation process with Eq. (37) to (39), we can obtain signal power, interference power and noise power

$$
\begin{aligned}
& P_{s}=\left|\mathbf{D}_{D}\right| \cdot{ }^{\wedge} 2+J_{\min } \cdot E\left(\frac{1}{\left|\widehat{h}^{u}\right|^{2}}\right) *\left(\left|\mathbf{D}_{D}\right| \cdot{ }^{\wedge} 2\right) * \operatorname{diag}\left(\left(\left|\mathbf{V}_{D}^{H}\right| \cdot{ }^{\wedge} 2\right) *\left(\left|\mathbf{V}_{D}\right| \cdot{ }^{\wedge} 2\right)\right), \\
& P_{\text {in }}=\left(1-\beta^{2}\right) \cdot E\left(\frac{1}{\left|h^{d}\right|^{2}}\right) \cdot\left(\left|\mathbf{D}_{D}\right| \cdot{ }^{\wedge} 2\right) *\left(\left(\left|\mathbf{V}_{D}^{H}\right| \cdot \wedge^{\prime} 2\right) *\left(\left|\mathbf{V}_{D}\right| \cdot{ }^{\wedge} 2\right)-\operatorname{diag}\left(\left(\left|\mathbf{V}_{D}^{H}\right| \cdot \wedge 2\right) *\left(\left|\mathbf{V}_{D}\right| \cdot \wedge 2\right)\right)\right),
\end{aligned}
$$

$$
P_{n}=\sigma_{n}^{2}
$$

After rigorous analysis, we know that Eq. (44), Eq. (45) and Eq. (46) are corresponding to $\left|e_{i, i}\right|^{2},\left|e_{i, j}\right|^{2}$ and $\sigma_{n}^{2}$ in Eq. (29). Substituting Eq. (44), Eq. (45) and Eq. (46) into Eq. (29), we can obtain the system capacity formula after 140 using the antenna calibration algorithm based on Wiener channel prediction in time-varying channel. 


\section{Simulation results and analysis}

In this section, we present the simulation results and analysis of the proposed method and compare it with the traditional antenna calibration method, and the validation of the results in 3.1 and 3.2 is to be provided by simulations. Antenna calibration in time-invariant or slow time-varying channel is called traditional antenna calibration technique for distinguishing with the proposed antenna calibration method based on channel prediction.

The simulation parameters of multi-antenna are shown in Table 1.

The simulation parameters of antenna mismatch are shown in Table 2.

Table 1: MIMO system simulation parameters

\begin{tabular}{|c|c|}
\hline Parameter & Value \\
\hline Number of subcarriers & 128 \\
\hline CP length & 16 \\
\hline Modulation & QPSK \\
\hline Doppler shift $(\mathrm{Hz})$ & $1,100,150,300$ \\
\hline Sampling time $(\mathrm{s})$ & $2.5 \mathrm{e}-6$ \\
\hline Number of antenna & $2 \times 2$ \\
\hline Channel & independent Rayleigh, frequency-flat \\
\hline
\end{tabular}

Table 2: Antenna mismatch simulation parameters

\begin{tabular}{|c|c|}
\hline Parameter & Value \\
\hline Amplitude mismatch $A$ & Lognormal distribution $10 \log _{10} A \sim N(0,2)$ \\
\hline Phase mismatch $\varphi$ & Uniform distribution $[-\pi / 6, \pi / 6]$ \\
\hline
\end{tabular}

150

Fig.2 shows the performance traditional antenna calibration based on SVD coding for MIMO system in time-invariant channel. The maximum Doppler shifts is 1 . We can see from the Fig.2 that the channel reciprocity loss caused by RF front-end can seriously deteriorate the performance of pre-equalization, 
while antenna calibration can well compensate for the loss, making pre-equalization work in ideal state. Fig.3 and Fig.4 show the BER and capacity of traditional

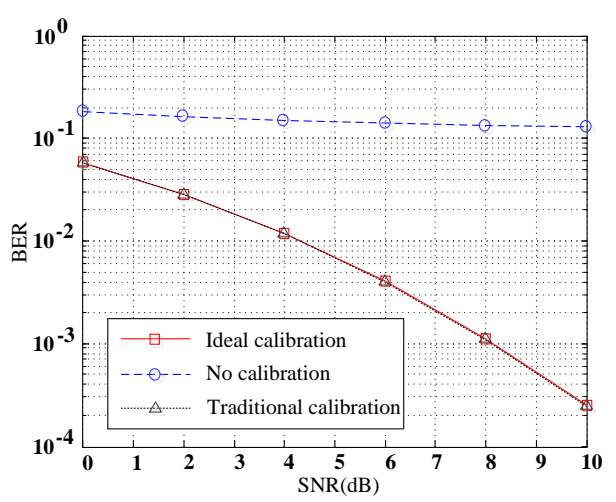

Figure 2: Performance of antenna calibration based on SVD coding for MIMO system.

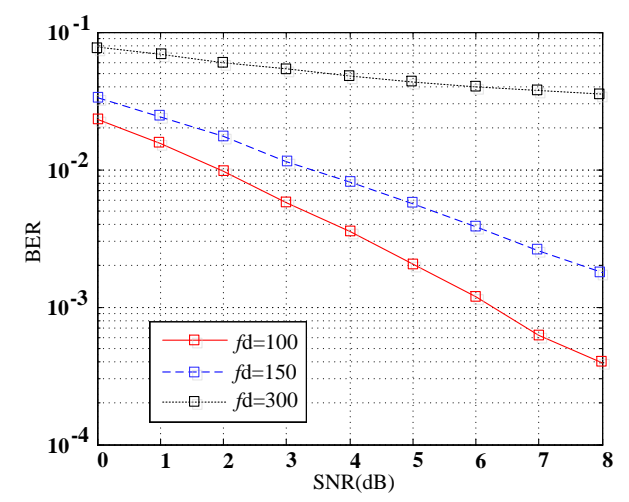

Figure 3: BER of traditional antenna calibration in time-varying channel.

antenna calibration for TDD-MIMO system in time-varying channel, respectively. In order to compare the performance in different time-variability, the maximum Doppler shifts in Fig.3 and Fig.4 is 100, 150 and 300, respectively. It is indicated by the figures that, with the enhancing of time-variability of channel, BER continues to increase and system capacity falls steadily, which proves that the time-variability leads to calibration error. Therefore, we can also know that traditional antenna calibration cannot effectively compensate for channel reciprocity loss due to time-variability, leading to the mismatch between SVD 


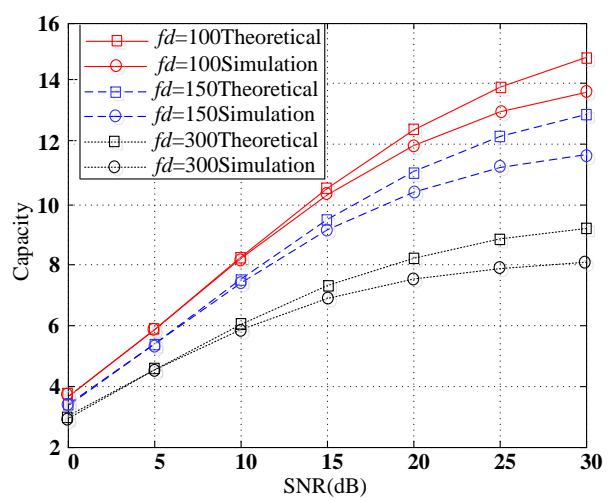

Figure 4: Capacity of traditional antenna calibration in time-varying channel.

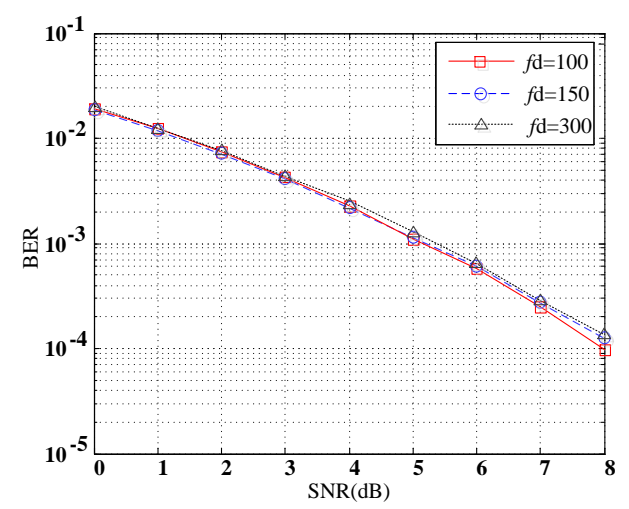

Figure 5: BER of antenna calibration based on channel prediction in time-varying channel.

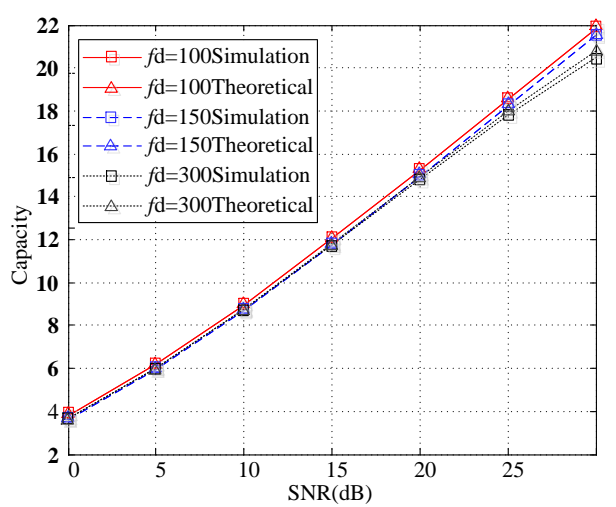

Figure 6: Capacity of antenna calibration based on channel prediction in time-varying channel. 

traditional antenna calibration is deteriorated.

Fig.5 and Fig.6 show the BER and capacity of antenna calibration based on Wiener channel prediction for TDD-MIMO system in time-varying channel, respectively. In order to compare the performance in different time-variability, It is indicated by comparison between Fig.3 and Fig.5 and comparison Fig.4 and Fig.6 that, the antenna calibration technique based on Wiener channel prediction can significantly reduce calibration errors caused by time-variability of channel, and then well compensate for the channel reciprocity loss, making the SVD pre-coding system work in the normal state.

\section{Conclusions}

The reciprocity loss caused by free-space channel and RF front-end can deteriorate the performance of pre-coding seriously for multi-antenna TDD system, and Antenna calibration can well compensate for the loss, making pre-coding work in ideal state. However, when channel changes dramatically, the free-space channel is no longer reciprocal, resulting in the loss of channel reciprocity and pre-coding. As a result, there is a large performance degradation when the traditional antenna calibration is used. Therefore, this paper proposes the antenna calibration technique based on Wiener channel prediction for TDD-MIMO system in time-varying channel, which can significantly reduce the calibration error due to time-variability. Through theoretical derivation, we get the capacity formula of the TDD-MIMO system using traditional antenna calibration and antenna calibration based on channel prediction. The theoretical analysis and simulation both show that the algorithm proposed in this paper can well compensate for the performance loss caused by time-variability and achieve better performance. 


\section{Acknowledgment}

The authors would like to thank the editors and the anonymous referees for their valuable comments and suggestions. This work was supported by the Research Foundation for Advanced Talents of Chengdu University of Information Technology (KYTZ201407), the National Science Foundation of China under Grant number 61471090, the Fundamental Research Funds for the Central Universities (Grant number ZYGX2013J112).

\section{References}

[1] Jia S. The think of B3G from the integration of broadband wireless access and broadband mobile communication technology $[\mathrm{M}]$. Telecommunications Science, 2006.6.

[2] Jia S, Suo S. 3GPP LTE technology principle and system design [M]. BeijingBeijing University of Posts and Telecommunications Press, 2008.11.

[3] Lilin D, Yue X, Wei N I, et al. Improved peak cancellation for PAPR reduction in OFDM systems[J]. IEICE transactions on communications, 2010, 93(1): 198-202.

[4] Xiexian Z. The fourth generation mobile communications based on TDD $[\mathrm{M}]$.Publishing house of electronics industry,2005.7

[5] Qualcomm Europe. Calibration procedures for TDD beam-forming[C]. R1080494, 3GPP TSG RAN WG1 meeting \#51bis, Sevilla, Spain, Jan. 14-18, 2008.

[6] Song Y S, Kwon D S. Computationally efficient compensations for some smart antenna systems[C]//Vehicular Technology Conference, 2004. VTC2004-Fall. 2004 IEEE 60th. IEEE, 2004, 7: 4786-4788.

[7] Mitsubishi Electric. Discussion on antenna calibration in TDD[C]. R1090563, 3GPP TSG RAN WG1 meeting \#56, Athens, Greece, Feb. 9-13, 2009. 
[8] Alcatel-Lucent Shanghai Bell, Alcatel-Lucent. Channel Reciprocity Modeling and Performance Evaluation[C]. R1-094622, 3GPP TSG RAN WG1 Meeting \#59, Jeju, Korea, 9th C 13th Nov. 2009.

[9] Hara Y, Yano Y, Kubo H. Antenna array calibration using frequency selection in OFDMA/TDD systems[C]//Global Telecommunications Conference, 2008. IEEE GLOBECOM 2008. IEEE. IEEE, 2008: 1-5.

[10] Guillaud M, Slock D T M, Knopp R. A practical method for wireless channel reciprocity exploitation through relative calibration[C]//ISSPA. 2005: 403-406.

[11] Kaltenberger F, Jiang H, Guillaud M, et al. Relative channel reciprocity calibration in mimo/tdd systems[C]//Future Network and Mobile Summit, 2010. IEEE, 2010: 1-10.

[12] Jian G., Yafeng W., Fan H., et al. Analysis on the impact of antenna gain mismatch on precoding vector[C]// Wireless Communications and Networking Conference (WCNC), 2011 IEEE. IEEE, 2011: 1794-1799.

[13] Fan H, Yafeng W, Jian G, et al. Antenna mismatch and calibration problem in coordinated multi-point transmission system[J]. IET communications, 2012, 6(3): 289-299.

[14] Shi J, Qinglin L, Mingli Y. An efficient method for enhancing TDD over the air reciprocity calibration $[\mathrm{C}] / /$ Wireless Communications and Networking Conference (WCNC), 2011 IEEE. IEEE, 2011: 339-344.

[15] Jian G, Chengkang P, Fan H, et al. Antenna gain mismatch calibration for cooperative base stations $[\mathrm{C}] / /$ Vehicular Technology Conference (VTC Fall), 2011 IEEE. IEEE, 2011: 1-5.

[16] Shengqian H, Liyan S, Chenyang Y, et al. Robust multiuser precoder for base station cooperative transmission with non-ideal channel reciprocity $[\mathrm{C}] / /$ Global Telecommunications Conference (GLOBECOM 2010), 2010 IEEE. IEEE, 2010: 1-5. 
[17] Kai Z, Zhisheng N. Multiuser MIMO downlink transmission over timevarying channels $[\mathrm{C}] / /$ Communications, 200\%. ICC'0\%. IEEE International Conference on. IEEE, 2007: 5514-5518.

[18] Lebrun G, Gao J, Faulkner M. MIMO transmission over a time-varying channel using SVD $[\mathrm{J}]$. Wireless Communications, IEEE Transactions on, 2005, 4(2): 757-764.

[19] Yue X, Xia L, Qingsong W, et al. A class of low complexity PTS techniques for PAPR reduction in OFDM systems[J]. Signal Processing Letters, IEEE, 2007, 14(10): 680-683.

[20] Li Z. Channel Reciprocity Compensation Method in Time-Varying TDDMIMO System Based on SVM Channel Prediction [Dissertation], Xian: XiDian University,2011.

[21] Dawei LShaoqian LYouxi T. Research on the performance of preequalization OFDM system [D]. Chengdu: UESTC.

[22] Proakis J G. Digital Communications (ISE) [J].2001.

[23] Yamin S, Xia L, Zhaofu K, et al. Antenna calibration using channel prediction for time-varying channels [J]. Journal of Modern Transportation, 2012, 20(4): 213-219. 